\title{
TOPOLOGICAL PROPERTIES OF THE CONTACT NETWORK OF GRANULAR MATERIALS
}

\author{
ROBERTO ARÉVALO, IKER ZURIGUEL and DIEGO MAZA \\ Departamento de Física, Facultad de Ciencias, \\ Universidad de Navarra, E-31080 Pamplona, Spain
}

Received March 14, 2008; Revised May 14, 2008

\begin{abstract}
The force networks of different granular ensembles are defined and their topological properties studied using the tools of complex networks. In particular, for each set of grains compressed in a square box, a force threshold is introduced that determines which contacts conform the network. Hence, the topological characteristics of the network are analyzed as a function of this parameter. The characterization of the structural features thus obtained, may be useful in the understanding of the macroscopic physical behavior exhibited by this class of media.
\end{abstract}

Keywords: Granular materials; force network; random graph.

\section{Introduction}

Granular materials are being widely studied by the physics community since they exhibit unusual and distinctive properties [Duran, 1999]. These materials are composed of macroscopic particles that interact by a dissipative contact force and can be thought of as displaying gas, liquid and solid phases. A suitable model for the study of granular materials is to consider each grain as a hard sphere, ignoring fragmentation and moving the effect of deformation to the dissipative term. As pointed in [Anikeenko et al., 2007], the wide applicability of these model to the study of liquids, glasses and colloids implies a paramount importance of the geometrical properties of the packing of hard spheres in determining the physics exhibited by the materials analyzed. The geometry of granular packing has been investigated i.e. by [Anikeenko et al., 2007] and [Aste et al., 2005] using Voronoi-Delaunay partitioning to identify structures in the former and volume distributions in the latter.

In the present work we propose, in the same line of those and other works [Ostojic et al., 2006], a structural study of granular packing but using tools specifically developed in the frame of complex networks. As will be explained later we define for each packing a network of contacts, see Fig. 1, of which the topological properties are studied afterwards.

The contact topology of a granular packing can be studied as a graph where particles are nodes and the interacting force pairs edges. This approach has important advantages. On the one hand, it is a quantitative tool as are not other ideas proposed in the granular community, namely, "force chains" [Peters et al., 2005]; it is an abstract point of view that allows to reach very primitive concepts such as connectivity over which to elaborate more complex definitions; and, finally, the field of complex networks provides us with a great amount of concepts and algorithms among which we can choose the most suitable for our proposes of characterization.

The remainder of this work is structured as follows: in Sec. 2 we explain the numerical method used and the protocol followed to obtain the samples that we study. In Sec. 3, the topological properties analyzed are defined and the results are presented for several different conditions. Finally, in Sec. 4 we summarize our results and draw some conclusions. 


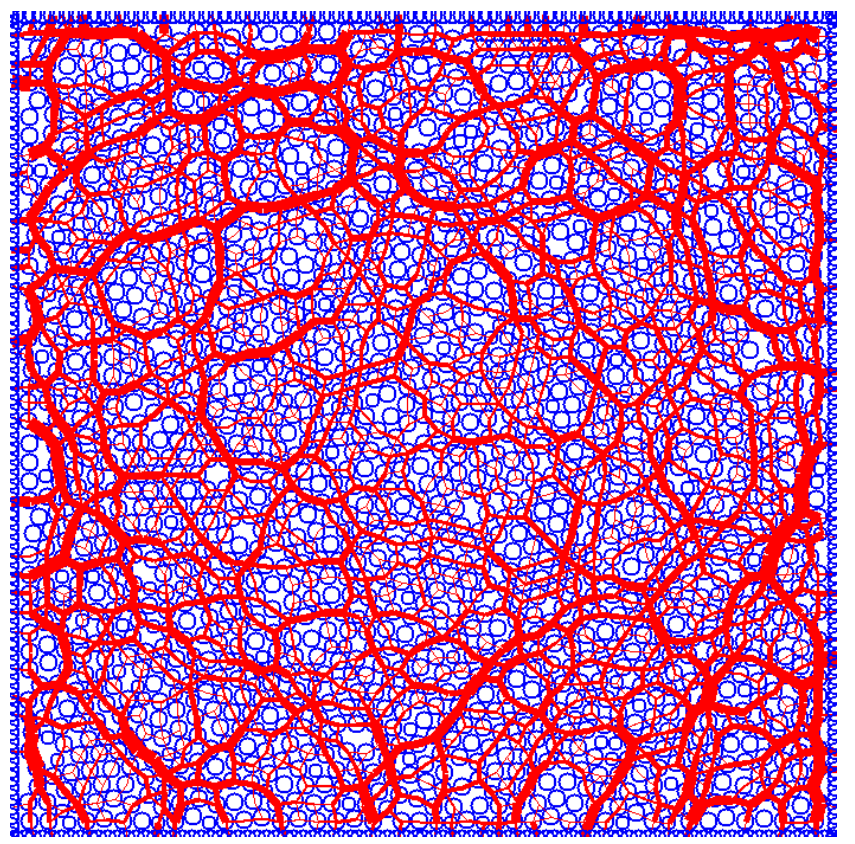

Fig. 1. Example of a compressed sample obtained with our simulations showing the force chains. The thickness of the lines represents the magnitude of the normal force originated by the interaction between each pair of particles.

\section{Numerical Method}

We perform soft particle molecular dynamics simulations of discs in two dimensions. The model of contact includes a linear restoring force in the normal direction of the impact and a tangential force providing static friction. The complete details of the simulation protocol are carefully described in [Arévalo et al., 2006]. The values used for the parameters of the force model are: the frictional coefficient $(\mu=0.5)$, the elastic constant $\left(k_{n}=\right.$ $10^{5}$ ), a dissipative coefficient $\gamma_{n}=150$, and the corresponding ones for the tangential component $\left(k_{s}=(2 / 7) k_{n}\right.$ and $\left.\gamma_{s}=300\right)$ with an integration time step $\delta=10^{-4} \tau$. The stiffness constants $k$ are measured in units of $m g / d$, the damping constants $\gamma$ in $m \sqrt{g / d}$ and time in $\sqrt{d / g}$. Here, $m, d$ and $g$ stand, respectively, for the mass of the discs, the diameter of the discs and the acceleration of gravity.

A typical simulation starts by randomly placing the discs in a wide horizontal area such that none of them is in contact with any other and the packing fraction is around 0.1. Discs are given random velocities drawn from a gaussian distribution. Four walls made up with the same grains that constitute the bulk compress the system until a certain predefined threshold of force is attained. It is important to note that due to the dissipative nature of the interactions, the final kinetic energy is vanishingly small. The final configuration obtained is saved in order to be carefully analyzed. This configuration is named a "jammed state" by the granular community and essentially corresponds to a metastable equilibrium state compatible with the history of the configuration.

We run simulations under several different conditions to check the variation of the results with the number of grains, polydispersity, friction coefficient, maximum applied pressure and geometry of the compression cell. Let us call sample A that obtained with a bidisperse mixture of discs, $15 \%$ with radii $d$ and the rest with radii $7 / 9 d$ and parameter values as given above; sample B has the same properties as A but the disks are monodisperse with radius $d$; sample $\mathrm{C}$ is bidisperse as $\mathrm{A}$ but the friction coefficient is $\mu=0.25$; sample $\mathrm{D}$ is the same as $\mathrm{C}$ but the final pressure is increased by $50 \%$; finally, sample $\mathrm{E}$ is the same as A but the boundary conditions are circular instead of square. For samples $\mathrm{A}$ and $\mathrm{B}$ we run simulations with 512, 1024 and 2048 discs, for samples C, D and E only with 2048 since, as will be shown, no significant dependence on system size is found. In order to attain good statistics we perform 20 independent simulations for each sample and average the results.

\section{Contact Network as a Complex Network}

In the first place we define our network, i.e. a set of nodes connected by edges [Newman, 2003], as follows. Every grain with, at least, one contact constitutes a node and edges are the connections between the grains (nodes) in contact. There is a contact between two grains if the distance between their center is smaller or equal to its diameter. A contact defines a certain amount of normal force $F$ between the grains. Such situations have been deeply studied by many authors using mainly lattice diffusive models [Liu et al., 2001]. One of the main results of these works is that, independently of the system details, the mean value of the force distribution $\langle F\rangle$ is a typical scale of the problem. Nevertheless, many questions remain open about the properties of these systems: why are the fluctuations in the force distribution as large as $\langle F\rangle$ ? Which statistical framework is suitable to explain the experimental results?

In order to study the role of the topology in this problem we will use the tools introduced in the theory of complex networks. We will introduce a force 
threshold $f$ such that any contact with a normal force bigger than $f$ is an edge, but contacts with lower values of normal force are not edges and grains with no contact are not nodes. Thus we, obtain a network which depends on $f$, and hence its topological properties can be studied as a function of $f$. In our definitions we do not consider the grains of the walls. In the remainder of this section, we present the results obtained for each of the topological properties studied along with their definitions [Newman, 2003; Costa et al., 2007].

\subsection{Connectivity}

In our case, the connectivity $k$ of a node represents the number of contacts between neighboring particles. Then, the degree distribution $P(k)$ is the distribution function of the number of contacts per particle. In Fig. 2(a) we show the degree distribution of sample A for three different sample sizes showing that there is no substantial variation. In all the cases, the maximum $P(k)$ is found for $k=3$ and around $95 \%$ of the particles present values of $k$ between 2 and 4 . The degree distribution for the rest of the samples with $N=2048$ is shown in Fig. 2(b). The overall behavior of the function $P(k)$ remains the same for all samples and only slight deviations are appreciated for samples $\mathrm{C}$ and $\mathrm{D}$. In particular, samples $\mathrm{C}$ and D display higher number of nodes with higher values of $k$. This result can be understood if it is considered that samples $\mathrm{C}$ and $\mathrm{D}$ are the ones with the smaller friction coefficient. This will result in a small amount of arches inside the sample and consequently a reduction of the amount of particles that display just two contacts.

In Fig. 3(a), we show the behavior of the average connectivity $\langle k\rangle$ as a function of the force threshold $f /\langle F\rangle$ for sample A. Again, this property seems independent of the system size. Figure $3(\mathrm{~b})$ shows the results obtained for the different samples. Again, small differences are appreciable for samples $\mathrm{C}$ and $\mathrm{D}$ without modification of the overall behavior.

The most prominent feature is a fast decay of the connectivity upon increasing the force threshold. It could be said that the small forces are the ones which keep the network connected and the connectivity almost disappears when they are removed.

\subsection{Geodesic distance and network diameter}

The geodesic distance $l$ between two nodes is the smallest number of edges that separate them. This quantity can be measured by a number of algorithms, we used the breadth first search. The diameter $D$ of the network is the longest of the geodesic distances. In Fig. 4, the normalized geodesic distance $l^{*}$ as a function of $f /\langle F\rangle$ is shown for sample A. The geodesic distance is normalized by $\sqrt{N} / 2$ since the geodesic distance increases with the number of particles conforming the sample $N$. This scaling of $l$ with the network size is what would correspond to a square lattice, so in the limit case of $f /\langle F\rangle \rightarrow 0$ our network seems to be not very different from a square one. The peak near $f /\langle F\rangle=1$ can

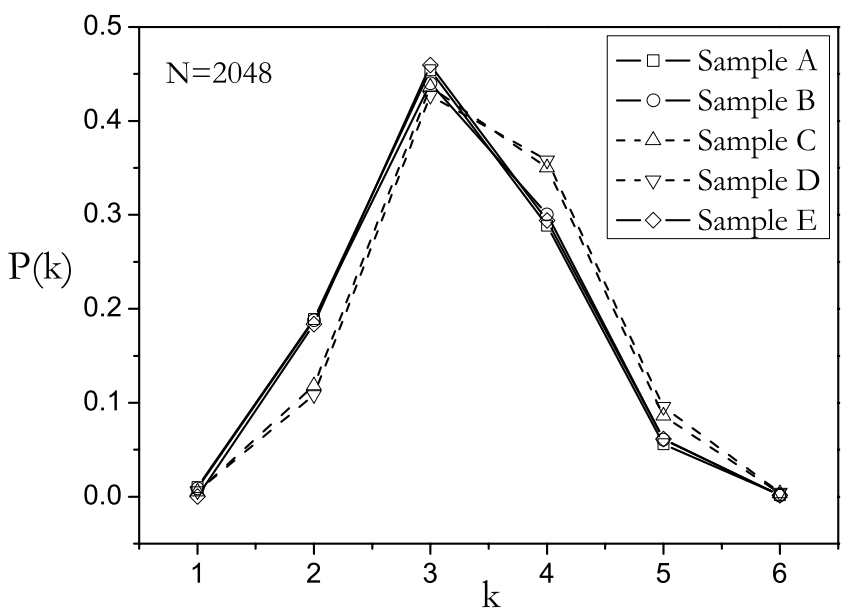

Fig. 2. Left panel shows $P(k)$ for sample A using $N=2048,1024,512$ discs. In the right panel we show $P(k)$ with $N=2048$ for all samples. 

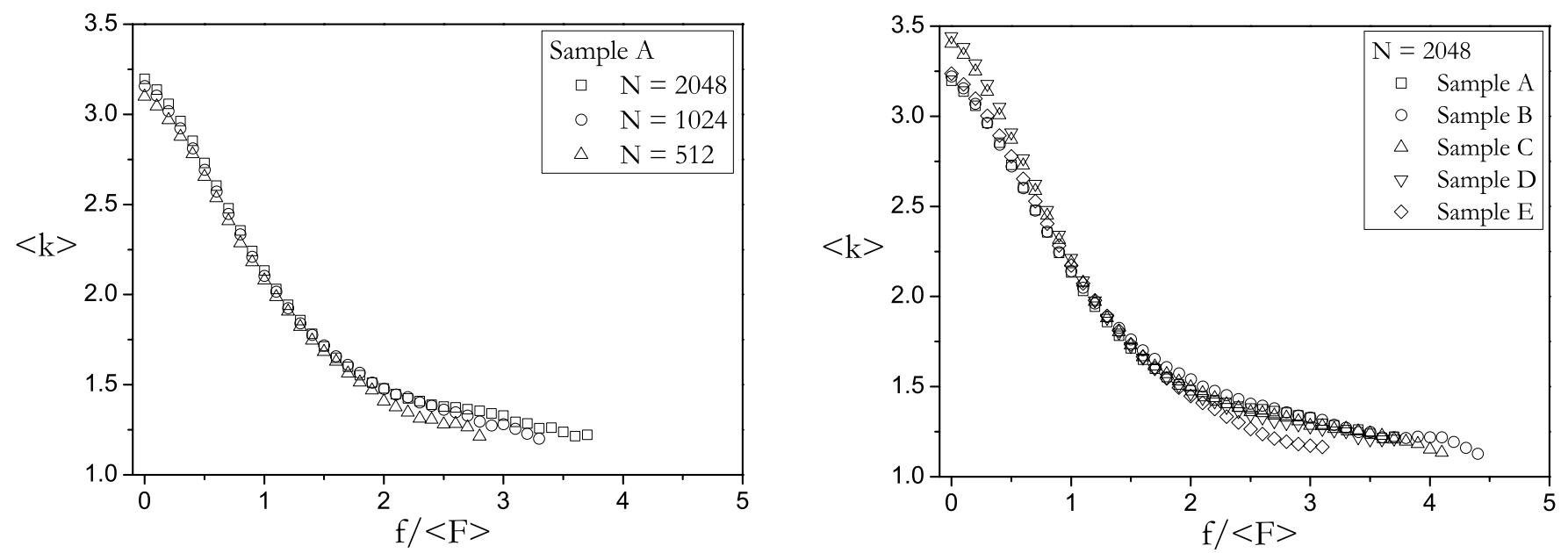

Fig. 3. (Left panel) Average connectivity of sample A. (Right panel) Average connectivity of all samples.
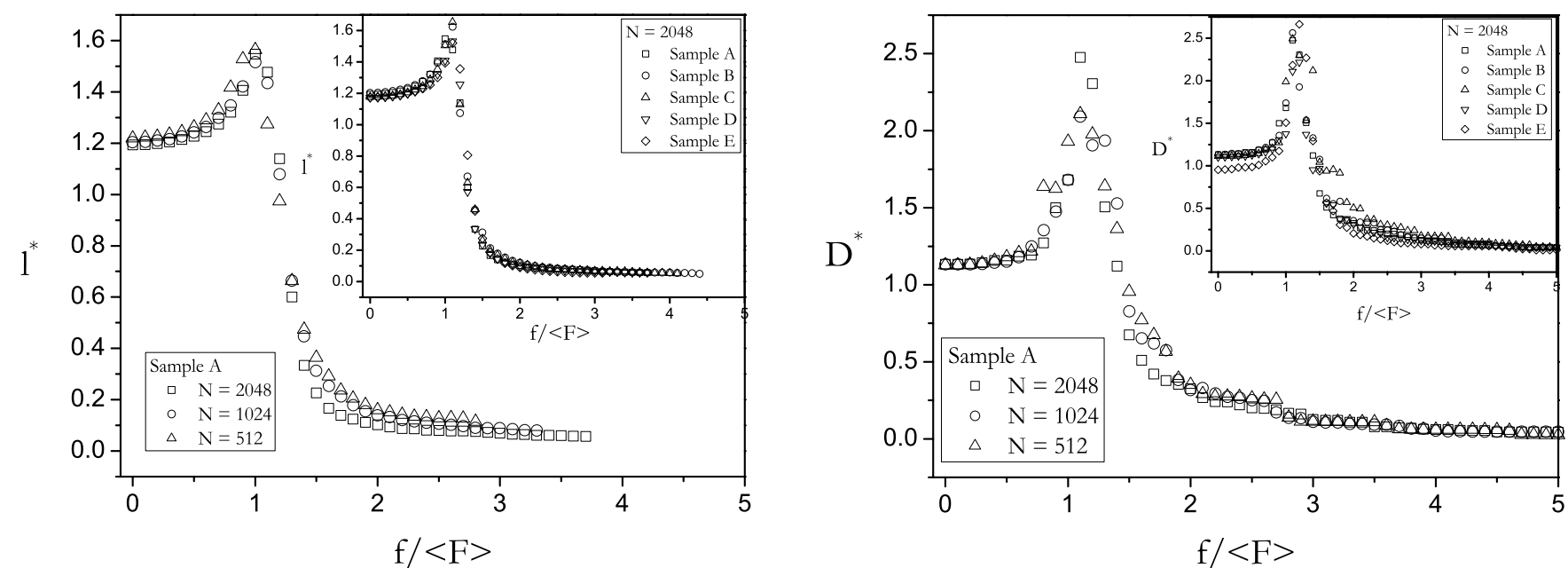

Fig. 4. (Left panel) $l^{*}$ is the geodesic distance $l$ normalized by $\sqrt{N} / 2$ for sample A. The inset shows $l^{*}$ for all the samples with $N=2048$. (Right panel) $D^{*}$ is the network diameter $D$ normalized by $\sqrt{2 N}$ for sample A. The inset shows $D^{*}$ for all the samples with $N=2048$.

be explained in terms of the polygons that appear in the network as will be shown later. In the inset of Fig. 4, it is shown that there is no difference in the behavior of the geodesic distance for the different samples.

The right panel of Fig. 4 shows $D^{*}$, the network diameter normalized by $\sqrt{2 N}$. This normalization factor is applied to show that the diameter of the network scales with the diagonal of the compression cell. The behavior is entirely similar to that of the geodesic distance and only a small deviation is noticeable for sample $\mathrm{E}$ which was generated with a circular cell. For this reason we can attribute this minor difference to the scaling factor.

\subsection{Number of nodes and maximum cluster size}

We define a cluster as a group of nodes mutually connected. The total number of nodes in the network includes nodes from different clusters. In the inset of Fig. 5(a) the total number of nodes is presented for sample A normalized by $N$, in semilogarithmic scale, showing that no variation appears upon increasing the network size. The inset of Fig. 5(b) shows the result for the rest of the samples. The number of nodes in the network decays exponentially, the line in both figures has slope 1.9, as the force threshold $f /\langle F\rangle$ is increased beyond $f /\langle F\rangle=1$. Before the point $f /\langle F\rangle=1$ the number 


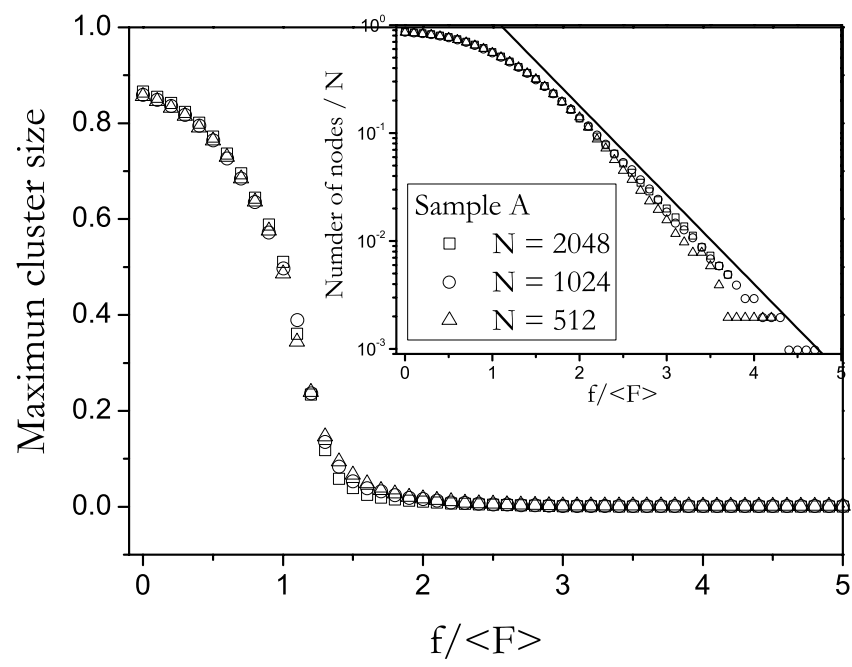

(a)

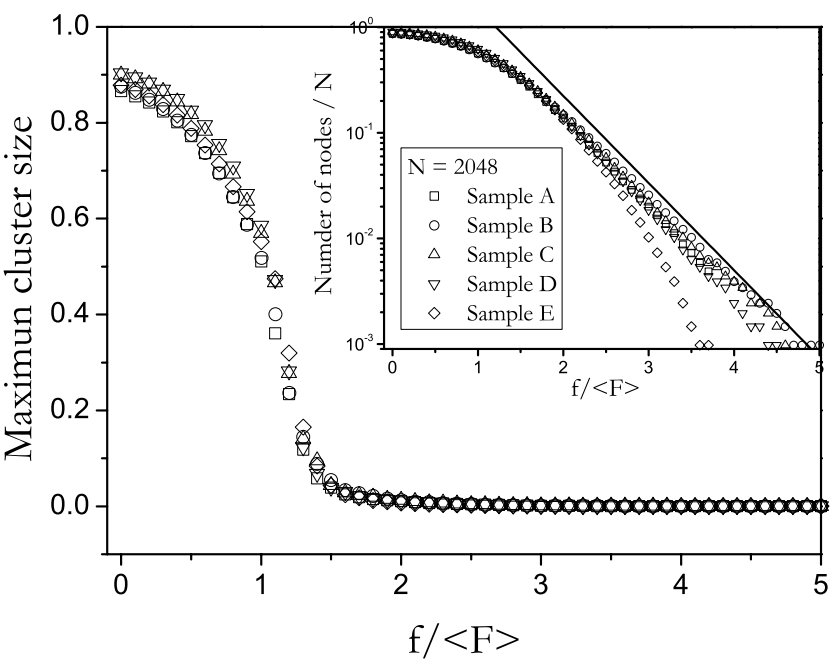

(b)

Fig. 5. (a) Size (in number of nodes normalized by $N$ ) of the largest cluster in the network for sample A. Inset: total number of nodes in the network normalized by $N$ for sample A with $N=512,1024,2048$. (b) Size (in number of nodes normalized by $N$ ) for all the samples and $N=2048$. Inset: total number of nodes for all the samples and $N=2048$. The line in both graphics has slope 1.9 .

of nodes decays only slightly. For $f /\langle F\rangle=0$ the normalized number of nodes is not 1 implying that there are grains without any contact. This is due to the frictional nature of the medium which is able to create arches surrounding one or more grains.

As $f /\langle F\rangle$ increases the network disaggregates in clusters that are not connected to each other. In Fig. 5(a) the size of the largest cluster, measured in number of grains, is shown for sample A normalized by $N$ while Fig. 5(b) shows this quantity for the rest of the samples. The largest cluster size dramatically drops in the vicinity of $f /\langle F\rangle=1$ and is almost zero beyond $f /\langle F\rangle \simeq 1.5$.

\subsection{Properties of clusters}

In this section, we further analyze the properties of clusters as defined in the previous section. As in percolation theory [Ostojic et al., 2006] we remove the largest cluster, which has been analyzed, and study the distribution of the sizes $s$ of the remaining clusters for different values of the force threshold: $n(s, f /\langle F\rangle)$. We use the samples with 2048. In Fig. 6 $n(s, 1.2)$, the cluster size distribution for $f /\langle F\rangle=$ 1.2 , is shown for all samples. In logarithmic scale, it can be fitted by a line whose slope, in this case is around 1.9. We have enough statistics only for values of $f /\langle F\rangle$ between 1 and 3 and in this range we find that the distribution of sizes behaves like $n(s, f /\langle F\rangle) \propto s^{\alpha}$ with $\alpha$ varying with $f /\langle F\rangle$.
In Fig. 7, we show the average cluster size $\langle s\rangle$ as a function of $f /\langle F\rangle$ with an inset showing the behavior of $\alpha$. If the largest cluster is retained to compute the size distribution, Fig. 7 displays a monotonically increasing function upon decreasing $f /\langle F\rangle$. Instead, a characteristic feature is revealed, a peak around $f /\langle F\rangle \simeq 1.2$ which is accompanied by a minimum in $\alpha(f /\langle F\rangle)$ around $f /\langle F\rangle=1.5$.

\subsection{Fractal dimension}

In the theory of critical phenomena the value of the fractal dimension determines the universality of a

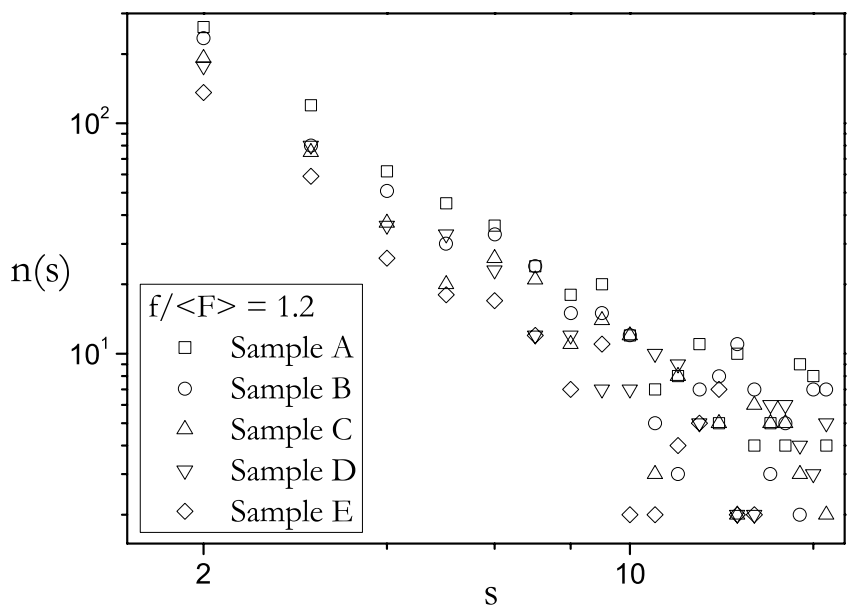

Fig. 6. The distribution of cluster sizes $s$ for all samples at $f /\langle F\rangle=1.2$ showing that it is a power function. 


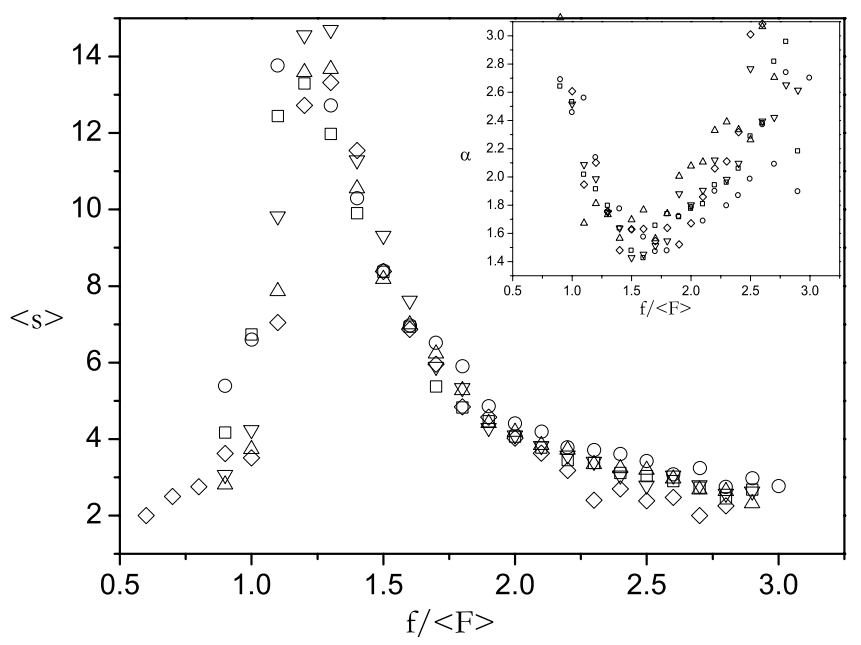

Fig. 7. Average cluster size, removing the largest one, as a function of the force threshold $f /\langle F\rangle$. The inset shows the power $\alpha$ of the cluster size distribution $n(s, f /\langle F\rangle)$ as a function of $f /\langle F\rangle$.

system, and thus, a set of properties. In this section, we compute the fractal dimension as a function of $f /\langle F\rangle$. Two such fractal dimensions can be defined [Song et al., 2005]: the mass fractal dimension and the box counting fractal dimension. The former is computed choosing a node and tracing circumferences of increasing radius $R$ around it. The mass $M$, in number of nodes, inside each circumference is computed and if it behaves as $M \propto R^{d_{M}}$ then $d_{M}$ is the mass fractal dimension. This procedure is repeated changing the initial node and averaging the results. The boxcounting fractal dimension is computed by analyzing how the minimum $N_{B}$ number of boxes necessary to cover the network changes with the box size $L$. If this verifies $N_{B} \propto L^{d_{B}}$ then $d_{B}$ is the box counting fractal dimension. The process of minimization involved in the last calculation renders it nonimmediate and so we have followed the methods exposed in [Song et al., 2007]. The results obtained for both, the mass fractal dimension and the box counting fractal dimension, are shown in Fig. 8 as a function of the force threshold $f /\langle F\rangle$.

Both dimensions are fairly equal to 1.8 for values of $f /\langle F\rangle$, roughly, lower than 1. A slight increase can be perceived from $f /\langle F\rangle=0$ until $f /\langle F\rangle \simeq 1$ where a marked drop takes place. This fall of the fractal dimension is sharper and deeper for the mass dimension but clearly present in both cases. The calculation of the fractal dimensions cannot be carried out beyond the limit shown since the network rapidly disaggregates. Thus, we find a change of behavior of the contact network in the vicinity of $f /\langle F\rangle=1$ that could be assigned to a change in the universality class that describes the network as a function of the force threshold.

\subsection{Third order loops of contacts}

A third order loop is defined as a three-step walk whose first and last nodes are the same. Third order loops are thus contacts arranged in a triangular fashion whose number can be computed by the clustering coefficient [Newman, 2003; Costa et al., 2007] or the third moment of the adjacency matrix [Goh et al., 2001]. In rigidity theory [Crapo, 1979] these
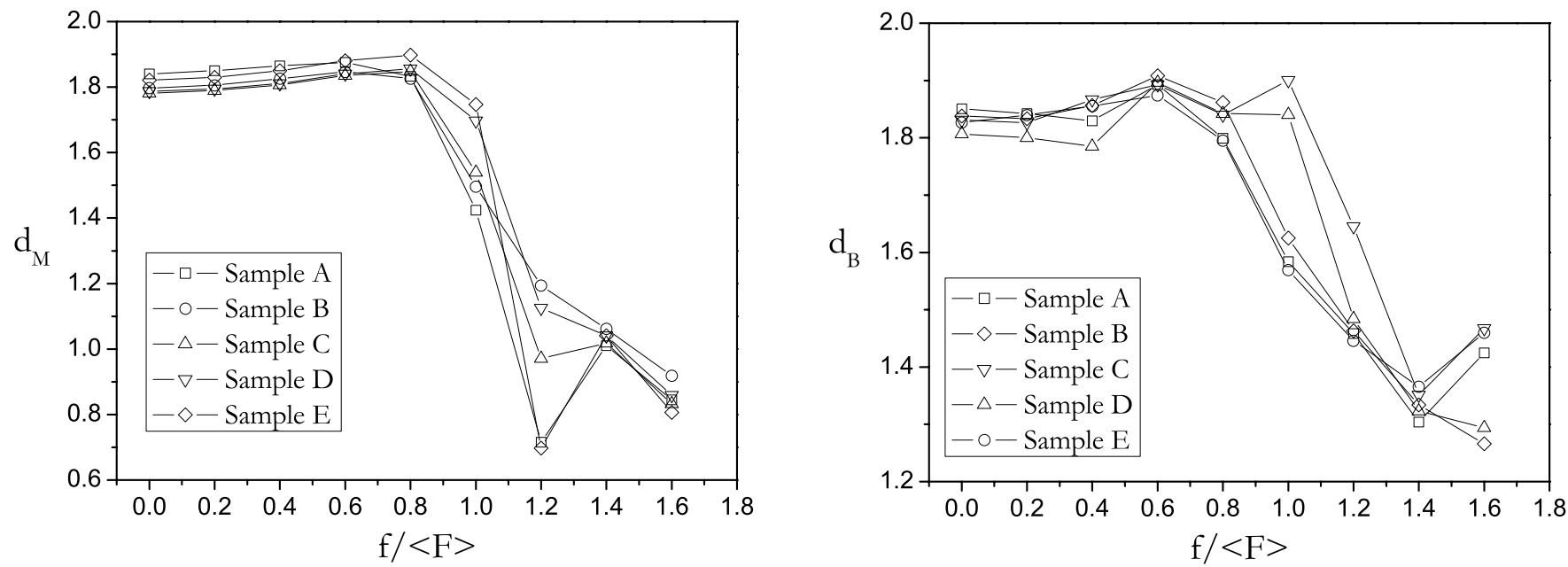

Fig. 8. (Left panel) The mass fractal dimension as a function of $f /\langle F\rangle$ for all samples with $N=2048$. (Right panel) The box counting fractal dimension as a function of $f /\langle F\rangle$ for all samples with $N=2048$. 
are, in two dimensions, the simplest rigid structures. Indeed if we consider a triangle whose edges are rigid and joined by freely rotational hinges, it will remain undeformed upon external perturbations. On the contrary, a square made of rigid edges and freely rotational hinges is easily deformed by shear on parallel sides. It is important to note that it is a sufficient, but not necessary, condition for a polygon to be rigid that all its faces are composed of triangles. Thus, triangles may be important for the rigidity displayed by granular packings in two dimensions.

In Fig. 9 the number of triangles is reported for all samples. There are no triangles beyond $f /\langle F\rangle \geq$ 1.5 and they are concentrated on the edges carrying a small amount of normal force, increasing exponentially when decreasing the force threshold. We believe that the apparition of third order loops of contacts is at the heart of the behavior found for some of the topological properties presented in this paper as the geodesic distance and the network diameter. For force values above $f /\langle F\rangle=1.5$ a decrease in the force threshold provokes a connection of different clusters in the network and then, both the geodesic distance and the network diameter grow. However, a further decrease in the force threshold below $f /\langle F\rangle=1.5$ implies the apparition of third order loops which will reduce the geodesic distance between the nodes that belong to them, and hence the diameter of the network. The way in which a third order loop reduces the geodesic distance between nodes can be easily understood. If

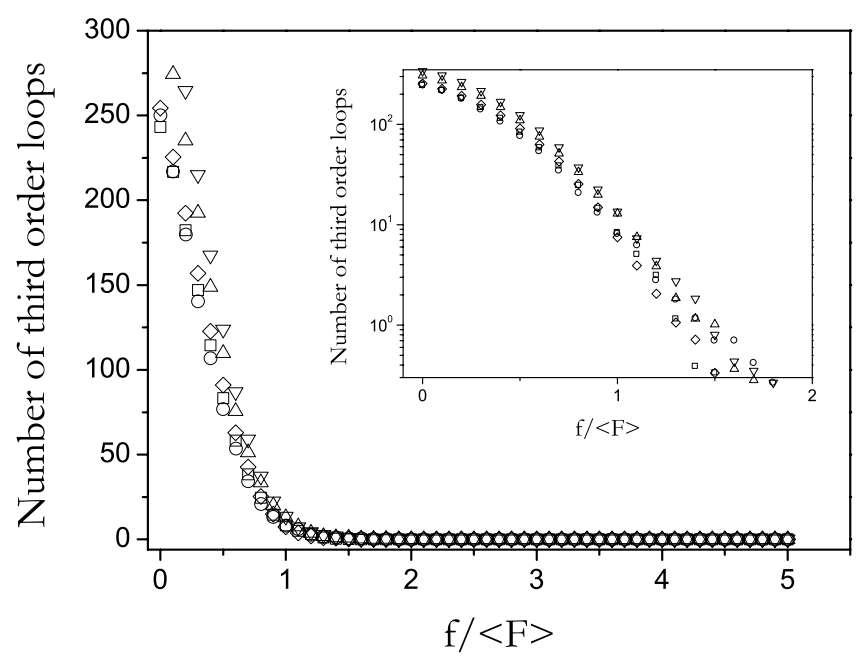

Fig. 9. Number of third order loops as a function of $f /\langle F\rangle$ for all samples with $N=2048$. The inset shows the same data in semilogarithmic scale. we imagine three nodes $(a, b, c)$ where the connections are $a-b$ and $b-c$, the mean geodesic distance in the cluster will be 1.33 as $a-b$ and $b-c$ are separated by one edge but $a-c$ are separated by two edges. In the same way, the diameter of this small network will be 2 as it is the maximum geodesic distance between the nodes. If now the nodes $a$ and $c$ are also connected giving rise to a third order loop, both the mean geodesic distance and the diameter of the network will be reduced to 1 .

\section{Discussion}

In this work, we report on some topological properties of force interactions in granular packings through ideas specifically introduced for complex networks. Our aim is to characterize the heterogeneity of these systems without using definitions that may change from one author to another, like usually seen in the case of force chains.

The set of properties analyzed is insensitive to the size of the system and shows only slight variations of behavior when the friction coefficient and the applied pressure are changed. Thus, they constitute a robust and useful description of a heterogeneous material like the packing studied. It is noteworthy that all the properties that have been analyzed as a function of the force threshold $f /\langle F\rangle$, that determines if an edge is present in the network or not, display some distinctive feature or sharp variation in the vicinity of $f /\langle F\rangle \simeq 1$. This behavior is indicative of a change in the structural properties of the network at this point. As has been pointed out by Radjai et al. there seems to be two subnetworks in the network of contacts, one "weak" network composed of small forces and containing around $60 \%$ of grains, and a "strong" network constituted by edges carrying a force above the average.

Our findings seem to support the existence of these two subnetworks. As the force threshold is increased we remove the weak network and retain only the strong one, leading to dramatic changes that signal the change of behavior expected if both subnetworks exist and are intrinsically different. In Fig. 10 we show the connectivities distribution for both subnetworks. It can be checked that they are quite similar for high connectivities while differ significantly around $k=2$.

A natural extension of this work is to consider the intensity of the force at every edge of the network and define weighted networks. This point of view could be a more suitable tool in order to relate 


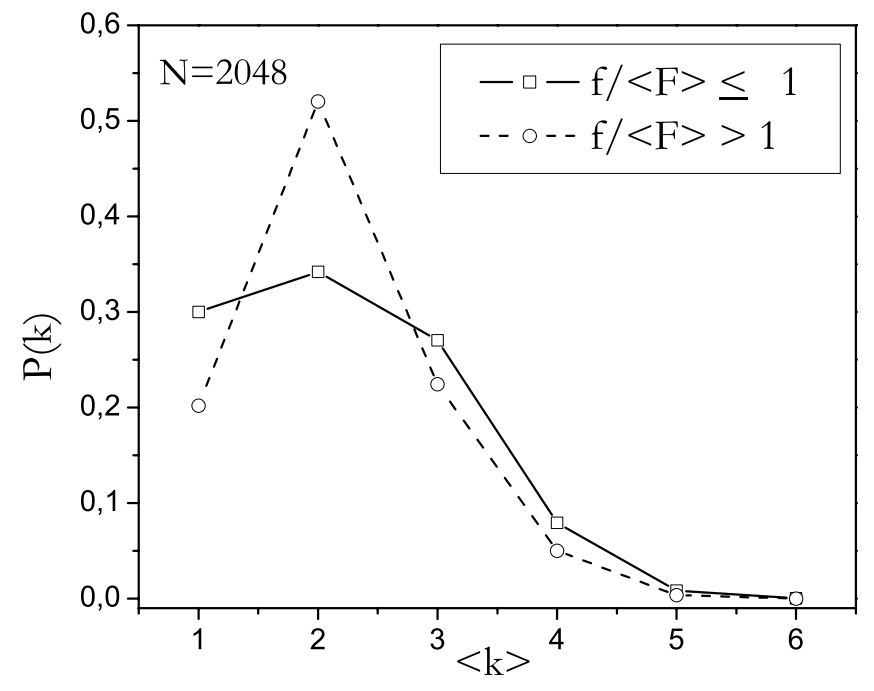

Fig. 10. Connectivities distribution for sample A where the nodes connected by a force $f /\langle F\rangle>1$ and $f /\langle F\rangle \leq 1$ have been treated separately. The subnetworks thus obtained exhibit well differentiated behaviors in the region of low connectivity.

structural features of the network with the physical properties of the packing; in particular, it could shed light into the question of the change of behavior at $f /\langle F\rangle \simeq 1$.

\section{Acknowledgments}

This work has been supported by project FIS200503881 (MEC, Spain), and PIUNA (University of Navarra). R. Arévalo thanks Friends of the University of Navarra for a scholarship.

\section{References}

Anikeenko, A. V. \& Medvedev, N. N. [2007] "Polytetrahedral nature of the dense disordered packings of hard spheres," Phys. Rev. Lett. 98, 235504.
Arévalo, R., Maza, D. \& Pugnaloni, L. A. [2006] "Identification of arches in two-dimensional granular packings," Phys. Rev. E 74, 021303.

Aste, T., Saadatfar, M. \& Senden, T. J. [2005] "Geometrical structure of disordered sphere packings," Phys. Rev. E 71, 061302.

Costa, L. da F., Rodrigues, F. A., Travieso, G. \& Boas, P. R. V. [2007] "Characterization of complex networks: A survey of measurements," Adv. Phys. 56, 167-242.

Crapo, H. [1979] "Structural Rigidity," Struct. Topol. 1, 26-45; Tay, T. S. \& Whiteley, W. [1985] "Generating isostatic frameworks," Struct. Topol. 11, 21-69.

Duran, J. [1999] Sands, Powders and Grains (SpringerVerlag, NY).

Goh, K. I., Kahng, B. \& Kim, D. [2001] "Universal behavior of load distribution in scale-free networks," Phys. Rev. E 64, 051903.

Liu, A. J. \& Nagel, S. [2001] Jamming and Rheology. Constrained Dynamics on Microscopic and Macroscopic Scales, eds. Liu, A. J. \& Nagel, S. (Taylor \& Francis, London and NY).

Newman, M. E. J. [2003] "The structure and function of complex networks," SIAM Rev. 45, 167.

Ostojic, S., Somfai, E. \& Nienhuis, B. [2006] "Scale invariance and universality of force networks in static granular matter," Nature 439, 828-830.

Peters, J. F., Muthuswamy, M., Wibowo, J. \& Tordesillas, A. [2005] "Characterization of force chains in granular material," Phys. Rev. E 72, 041307.

Radjai, F., Jean, M., Moreau, J.-J. \& Roux, S. [1996] "Force distributions in dense two-dimensional granular systems," Phys. Rev. Lett. 77, 274-277.

Song, C., Havlin, S. \& Makse, H. A. [2005] "Selfsimilarity of complex networks," Nature 433, 392395.

Song, C., Gallos, L. A., Havlin, S. \& Makse, H. A. [2007] "How to calculate the fractal dimension of a complex network: The box covering algorithm," J. Stat. Mech. 3, P03006. 